INPLASY

PROTOCOL

To cite: Hui et al. The comparison of targeted fusion biopsy and systematic biopsy for the diagnosis of prostate cancer: A protocol for systematic review and meta analysis. Inplasy protocol 202180017. doi: 10.37766/inplasy2021.8.0017

Received: 04 August 2021

Published: 04 August 2021

Corresponding author: Cong Wang

wc027214@163.com

Author Affiliation:

The First Affiliated Hospital of Dalian Medical University

Support: LZ2020027.

Review Stage at time of this submission: Preliminary searches.

Conflicts of interest: None declared.

\section{The comparison of targeted fusion biopsy and systematic biopsy for the diagnosis of prostate cancer: A protocol for systematic review and meta analysis}

Hui, J1; Cong, W2

Review question / Objective: The present meta-analysis aimed at determining the accuracy of targeted fusion biopsy of prostate cancer detection compared with that of systematic biopsy.

Condition being studied: Studies suggested targeted fusion biopsy of mpMRI and TRUS is performed in men suspected of prostate cancer to optimize the detection of clinically significant disease, while reducing the burden of biopsies. Information sources: PubMed, Web of Science, Cochrane Library, and Chinese biomedical databases will be searched from their inceptions to the August 1st, 2021. We will not impose any limitations to language and publication status. The search strategy will be built with the assistance of a professional librarian.

INPLASY registration number: This protocol was registered with the International Platform of Registered Systematic Review and Meta-Analysis Protocols (INPLASY) on 04 August 2021 and was last updated on 04 August 2021 (registration number INPLASY202180017).

\section{INTRODUCTION}

Review question / Objective: The present meta-analysis aimed at determining the accuracy of targeted fusion biopsy of prostate cancer detection compared with that of systematic biopsy.
Condition being studied: Studies suggested targeted fusion biopsy of mpMRI and TRUS is performed in men suspected of prostate cancer to optimize the detection of clinically significant disease, while reducing the burden of biopsies. 


\section{METHODS}

Participant or population: The patients should be men who undergone prostate biopsy.

Intervention: Fusion biopsy.

Comparator: Systematic biopsy.

Study designs to be included: This study included high quality randomized controlled trials, cohort studies or casecontrol studies.

Eligibility criteria: 2.1.1. Type of study. This study included high quality randomized controlled trials, cohort studies or casecontrol studies.2.1.2. Type of patients. The patients should be men who undergone prostate biopsy. We will not apply any restrictions of race, age, education background, and economic status.2.1.3. Intervention and comparison. This study compared fusion biopsy with systematic biopsy for PCa.2.1.4. Type of outcomes. The primary outcome was detection rates of PCa.

Information sources: PubMed, Web of Science, Cochrane Library, and Chinese biomedical databases will be searched from their inceptions to the August 1st, 2021. We will not impose any limitations to language and publication status. The search strategy will be built with the assistance of a professional librarian.

Main outcome(s): The primary outcome was detection rates of $\mathrm{PCa}$.

Quality assessment / Risk of bias analysis: The quality of the primary studies was assessed using the Cochrane risk of bias tool[8] by two independent researchers and an additional investigator in the case of any conflicts.

Strategy of data synthesis: We calculated the pooled summary OR and its $95 \%$ confidence interval(Cl). The Cochran's Qstatistic and 12 test were used to evaluate potential heterogeneity between studies. If $Q$ test shows a $\mathrm{P} 50 \%$ which indicates significant heterogeneity, the randomeffect model was conducted, or else the fixed-effects model was used.

Subgroup analysis: If necessary, subgroup analysis will be performed.

Sensitivity analysis: In order to evaluate the influence of single study on the overall estimate, sensitivity analysis was performed.

Country(ies) involved: China.

Keywords: meta-analysis; prostate cancer; biopsy.

Contributions of each author:

Author 1 - Jin Hui.

Author 2 - Cong Wang. 